\title{
Bacterial infectious disease control by vaccine development
}

\author{
Roy Curtiss III \\ Department of Biology, Washington University, St. Louis, Missouri, USA \\ J. Clin. Invest. 110:1061-1066 (2002). doi:10.1172/JCI200216941.
}

There is a need to develop vaccines with the potential for global use against bacterial infectious diseases. These should be inexpensive to produce, stable in the absence of refrigeration, safe and efficacious, and able to be given orally or intranasally rather than by injection. Here, I will focus on efforts to develop live attenuated bacterial vaccines and the use of recombinant attenuated bacteria to induce protective immunity against heterologous bacterial pathogens. I will not discuss bacterial vaccine vectors expressing viral, parasite, or fungal antigens, since lack of posttranslational modification and improper folding generally lead to induction of immune responses that are not protective, although induction of cell-mediated immunity can often be protective. Also omitted will be a discussion of the use of attenuated bacterial vaccine vectors for the delivery of DNA vaccines, wherein problems of processing of viral, parasite, and fungal antigens in bacteria are rectified by the synthesis and modification of these antigens within the eukaryotic cell. Lastly, although I acknowledge their value, especially in the developed world, I will not dwell on progress in the development of subunit and conjugate vaccines. Studies of such vaccines, however, often provide valuable information on identification and characterization of protective antigens that can be expressed in live recombinant attenuated bacterial vaccines of the kind I consider below.

\section{Attributes of safe and effective live bacterial vaccines}

The bacterial vaccine or antigen delivery vector. Attenuation should be sufficient to decrease if not eliminate induction of undesirable disease symptoms. In this regard, the nutritional status and health of the population to be vaccinated should be considered. The attenuation should be an inherent property of the bacterial vaccine

\footnotetext{
Note: This article is part of the Perspective Series "The Future of vaccine design", edited by Peter Palese and Adolfo García-Sastre, published in issues $1-3$ of volume 110 . Due to a production error, its publication was delayed. We regret this error.

Address correspondence to: Roy Curtiss III, Department of Biology, Washington University, 1 Brookings Drive, Campus Box 1137, St. Louis, Missouri 63130-4899, USA. Phone: (314) 935-6819; Fax: (314) 935-7246; E-mail: rcurtiss@biology.wustl.edu.

Conflict of interest declared: No conflict of interest has been declared. Nonstandard abbreviations used: gut-associated lymphoid tissue (GALT); Bacillus Calmette-Guérin (BCG).
}

and not be dependent on fully functional host defenses and immune response capabilities. The attenuation should not be reversible by diet or by host modification of diet constituents, including by host-resident microbial flora. The attenuation should not lead to the development of a persistent carrier state for the vaccine. However, it is conceivable that there may be situations in which a persisting live vaccine could be beneficial. The attenuated vaccine should be sufficiently invasive and persistent to stimulate both strong primary and lasting memory immune responses.

A vaccine should also be designed to minimize tissue damage that is not needed to induce an effective immune response. For example, vaccines for enteric pathogens must access the gut-associated lymphoid tissue (GALT) via invasion and transcytosis through $M$ cells. However, attachment to and invasion into enterocytes can lead to undesirable diarrheal episodes that do not contribute to the desired immune response. As even attenuated vaccine strains may cause disease in a few unlucky individuals, safety considerations dictate that any live bacterial vaccine should be susceptible to all clinically useful antibiotics. Lastly, the attenuated vaccine should possess some containment features to reduce its shedding and/or survival in nature to preclude vaccination of individuals who did not elect to be vaccinated. However, persistence of a live vaccine with potential for individual-to-individual spread could have a positive public health benefit, as is probably the case for vaccination against polio.

The plasmid vector component. Stability of protective antigen expression in vivo is essential for recombinant vaccine efficacy. Insertion of genes into the chromosome can increase stability, but the level of antigen expression is generally too low to stimulate an adequate immune response. Since the level of protein synthesis in bacteria is very much dependent upon gene copy number, antigen production can be vastly increased by use of multicopy plasmid vectors. In this case, the use of a balanced-lethal host-vector system wherein the plasmid possesses a gene complementing a chromosomal deletion mutation of a vital gene, such as for cell wall synthesis or DNA stability or replication, ensures that the plasmid is maintained (1). An alternate approach to ensuring retention of the plasmid vector is the "HokSok" strategy, which also results in bacterial cell death if the plasmid is lost (2). When a plasmid vector with 
high copy number is used, the level of expression of the gene encoding the vector-selective marker can be far in excess of that necessary for maintenance of the vector. In these instances, overexpression of such a gene product further attenuates the vaccine, presumably due to the added energy drain on the recombinant vaccine. To address this problem, the selective marker gene can be designed to include a ribosome-binding recognition sequence but no promoter (3). As a safety consideration, it is desirable that plasmid vectors possess some containment features to minimize the possibility of transfer to and maintenance in other bacterial species. The vector should therefore be nonconjugative, should preferably be nonmobilizable, should possess a narrow replicon host range, and should not specify resistance to any antibiotic.

In general, the level of antigen synthesis can be lower when a Th1 cell-mediated immunity-type (CMI-type) response is desired but must be much higher if Th2 mucosal and systemic antibody responses are necessary. Promoters driving the expression of foreign antigens have often been constitutive (4), but constant antigen synthesis may decrease vaccine fitness to result in increased attenuation and decreased immunogenicity. Improved results have frequently been observed by use of the nirB (5), pagC (6), and $d m s A$ (7) Salmonella promoters that initiate transcription at higher levels in vivo. Antigen expression can also be delayed until after the vaccine has invaded lymphoid tissues by use of a regulatory cascade with araC $\mathrm{P}_{\mathrm{BAD}}$-controlled expression (8) of a repressor whose synthesis is dependent on the presence of arabinose during in vitro cultivation. In this case, the antigen gene is coupled to a promoter with transcription repressed by the araC $\mathrm{P}_{\mathrm{BAD}}$-regulated repressor. Since there is no free arabinose in vertebrate tissues, the foreign antigen gene is eventually de-repressed in vivo as the repressor is diluted out as a consequence of vaccine cell division.

The immune response to a protective antigen can be influenced by the location of the antigen. Although good immune responses have been observed for antigens retained in the cytoplasm or secreted into the periplasm of Gram-negative vaccines (9), placement on the surface or secretion into the supernatant fluid making use of heterologous (10) or homologous (11) secretion mechanisms can further enhance the level and type of immune response induced. In addition, by employing the type III secretion apparatus of Salmonella and Yersinia, antigens with $\mathrm{T}$ cell epitopes can be delivered into the cytoplasm of antigen-presenting cells within the immunized eukaryotic host, resulting in a CD8-restricted CTL response (12).

The host-vector combination. The synthesis of pathogen antigens by recombinant attenuated host-vector antigen delivery systems can contribute to further attenuation to reduce immunogenicity (13), another important consideration in vaccine design. To enhance the likelihood of sufficient colonization and persistence in lymphoid tissues to stimulate protective immunity, the growth properties of the vaccine construct should match those of the host-vector control not expressing the antigen. The recombinant system should also be designed to maximize the immune response to the foreign antigen(s) and minimize the competing immune responses to bacterial vector antigens.

\section{Attenuated bacterial vaccines}

Mycobacterium bovis Bacillus Calmette-Guérin (BCG), a vaccine to prevent Mycobacterium tuberculosis infection, was the first and continues to be the most widely used live bacterial vaccine in the world. Nevertheless, $M$. tuberculosis infects one-third of the human population and causes 2 million deaths each year. The strains of BCG used as vaccines have recently been carefully characterized (14) and shown to lack expression of certain antigens present in $M$. tuberculosis that might be important for protective immunity. The means of achieving attenuation by repetitive culture passage likely led to a number of genetic changes that not only contribute to attenuation but also reduce immunogenicity. Since M. tuberculosis infection often results in a carrier state with reactivation many years later, it is critical, in the development of new live, attenuated M. tuberculosis vaccine candidates, that one preclude the creation of a vaccine that can be as persistent in a hidden form as $M$. tuberculosis is.

Salmonella typhi Ty21a is a widely used licensed live vaccine that is exceedingly safe but requires three to four immunizations to induce long-term (at least 6-7 years) protective immunity in two-thirds of individuals immunized (15). Newer, more immunogenic, live $S$. typhi vaccines are currently being clinically evaluated with the goal of developing an attenuated S. typhi vaccine that can induce protective immunity in a high percentage of individuals with a single dose. This effort has been hampered by the fact that most means of attenuation, with one exception ( $p h o P Q$ ), that were effective in attenuating Salmonella typhimurium and rendering it highly immunogenic in mice fail to attenuate $S$. typhi sufficiently in humans $(16,17)$. For example, S. typhi strains with double defined deletion mutations in aro genes that possess requirements for a number of vitamin cofactors theoretically unavailable in vertebrates. Such strains should therefore be unable to grow for more than seven to ten generations in vivo. Nevertheless, positive blood cultures with fevers are observed when high doses of the double aro vaccine are administered to human volunteers (18). This unacceptable level of reactogenicity has been corrected by introduction of an additional mutation, $b t r A$, eliminating a heat shock protease (19), which confers additional attenuation without sacrificing too much immunogenicity (but see ref. 20). Two such double aro htrA vaccine candidates are being clinically evaluated (21). Ty800, which is very likely the best-attenuated $S$. typhi vaccine candidate, has a defined deletion mutation of the two-component virulence-regulating genes, phoPQ. In human trials, Ty800 induced high-level immune responses with a single dose and without adverse side effects, except for diarrhea in a small number of individuals (22). 
The live attenuated Bacillus anthracis strain Sterne, licensed as a veterinary vaccine, was used for human immunization in the former Soviet Union but was too reactogenic (23). New, improved, live attenuated $B$. anthracis vaccines have given promising results in inducing protective immunity in guinea pigs $(24,25)$ but have yet to undergo clinical evaluation in humans.

Development of a live attenuated Shigella vaccine that is sufficiently attenuated to be nonreactogenic yet adequately invasive to be highly immunogenic has been attempted for over 30 years. Due to substantial progress in understanding the molecular genetic basis of virulence of Shigella since the early 1980s, there are now two candidate vaccines with defined attenuating deletion mutations that induce protective immunity to Shigella challenge in humans without severe associated diarrheal symptoms $(26,27)$. Additional clinical trials on these vaccines are likely. Nevertheless, most vaccine development work has been with specific Shigella flexneri serotypes (of which there are 12), and serotype-specific immunity is important. In addition, severe dysentery is caused by Shigella dysenteriae and less severe but more prevalent dysentery by $S$. sonnei and S. boydii. Development of effective vaccines to prevent the global Shigella infections of 1 million or more each year, caused by some 38 serotypes, will therefore not be easily achieved.

Two live attenuated Vibrio cholera vaccine candidates have been constructed and clinically evaluated, and one, CVD 103-HgR, is now commercially available (28). Both of these vaccines possess defined deletion mutations that preclude the ability to express cholera toxin as well as some of the other accessory toxins that contribute somewhat to the diarrhea caused by $V$. cholera infection. Although much effort has gone into the design of these strains, it is difficult to see how these noninvasive candidate vaccines will induce a long-term mucosal or systemic immune memory. The very fact that the vaccine strains must persist in the intestinal tract to stimulate an immune response, and that they do so by the expression of toxin-coregulated pili, indicates that some diarrhea will result from immunization, since nontoxigenic strains of bacteria that possess the ability to express the pilus adhesins necessary for colonization can still cause significant diarrhea and sometimes death in animals (29).

\section{Attenuated recombinant bacterial host-vector systems}

Strains of S. typhimurium and S. typhi were first used as recombinant vectors for antigen delivery $(4,9)$, and BCG was later developed as an antigen delivery vector (30). Most recently, attenuated derivatives of S. flexneri, Listeria monocytogenes, and B. anthracis are being evaluated as recombinant antigen delivery vaccines.

Over 15 years of research, with a substantial and ever-increasing pace of published information, explores the use of attenuated $S$. typhimurium and $S$. typhi antigen delivery vaccines. Although recombinant attenuated $S$. typhimurium strains expressing a diversity of antigens from various types of pathogens have often induced protective immunity in mice and a number of agriculturally important animals, recombinant attenuated $S$. typhi Ty2 strains evaluated in human volunteers have induced diminished immune responses to the Salmonella vector and insignificant or nonexistent immune responses to expressed antigens from several different pathogens (31-33). Although there are several possible explanations, one significant problem may be the choice of the S. typhi antigen delivery strain, Ty2. We have demonstrated in S. typhimuri$u m$ that chromosomal RpoS-regulated genes are necessary for invasion into and colonization of $\mathrm{M}$ cells overlying the GALT (34). In accord with this, rpos mutants of $S$. typhimurium, with or without the virulence plasmid that possesses RpoS-regulated genes that play no role in colonization of the GALT, exhibit diminished immunogenicity, an observation confirmed by others (35). We demonstrated the same defect in dependence on RpoS-regulated chromosomal genes for effective colonization of lymphoid tissues, presumably the nasal-associated lymphoid tissue and bronchial-associated lymphoid tissue, following intranasal immunization in mice. Unfortunately, $S$. typhi Ty2, which was isolated in 1918 and maintained in the laboratory ever since, possesses an rpos allelic defect that is also present in the not-so-immunogenic licensed $S$. typhi Ty21a vaccine. It is known that $R p o S^{+}$ S. typhi strains are more virulent for human volunteers than are the $S$. typhi Ty 2 derivatives attenuated by the same mutations (18). Use of $R p o S^{+} S$. typhi antigen delivery vectors will therefore necessitate degrees of attenuation in excess of what is necessary to attenuate S. typhi Ty2 strains for humans, but these means of attenuation must not compromise immunogenicity.

Despite these setbacks, results with recombinant attenuated $S$. typhimurium vaccines in animals suggest that recombinant attenuated $S$. typhi vaccines could be developed to protect against infections due to Hepatitis B virus (HBV), papillomaviruses, Streptococcus pneumoniae, Helicobacter pylori, Yersinia pestis, human enterotoxigenic and Escherichia coli strains, M. tuberculosis, $L$. monocytogenes, B. anthracis, Clostridium tetani, and Clostridium difficile. In addition, some progress is being made in developing vaccines to protect against infections due to Chlamydia, Rickettsia, Francisella tularensis, Neisseria meningitidis, Brucella, and Porphyromonas. One of the more tantalizing challenges is to develop a recombinant attenuated multivalent vaccine that would substitute for the injectable DPT vaccines (36). Fragment C of C. tetani has been expressed in attenuated Salmonella in mice, using a codon-optimized sequence and induced protective immunity to $C$. tetani toxin challenge. It is the only antigen delivered by an attenuated S. typhi Ty2 strain to induce a modest level of immunity in human volunteers (37). Research on expression of the Corynebacterium diphtheriae CRM 197 in S. typhimurium and S. typhi looks encouraging (38). A number of Bordetella pertussis protective antigens have been expressed in recombinant attenuated S. typhimurium, and significant immune responses in mice have been observed. Certainly, a multivalent recombinant attenuated $S$. typhi expressing all of these antigens would result in a 
vaccine that could be administered orally or intranasally and would do much to reduce mortality due to these pathogens in the developing world.

Since phagocytic cells do not readily lyse mycobacterial cells, BCG or other attenuated mycobacterial strains do not seem promising as antigen delivery vectors unless the expressed antigens can be secreted from the cells. In the case of protective antigens from Borrelia burgdorferi, this approach has successfully induced protection in mice, but it failed to induce antibody responses to the Borrelia OspA antigen in human volunteers (39). Secretion of M. tuberculosis antigens by BCG to induce protection against $M$. tuberculosis challenge in guinea pigs has also been achieved (40). Use of recombinant attenuated $L$. monocytogenes (41), $B$. anthracis (42), and S. flexneri (43) as antigen delivery vectors is hampered by lack of a suitable animal host for developmental research, the reliance on results with injected candidate recombinant vaccines, and/or the need to develop strains that are attenuated, nonreactogenic, and highly immunogenic in human volunteers.

\section{Antigen selection}

Identification of protective antigens of a pathogen to express in a recombinant attenuated bacterial vaccine is not an easy task. Presence of antibodies in a host surviving infection can offer clues. However, a high-level antibody response does not always correlate with protection. Of course, if one can obtain an $\mathrm{mAb}$ to an antigen that can passively protect against the pathogen, that is a good sign that that antigen is likely to induce a protective response. Similarly, the identification of antigen-specific $T$ cell populations whose passive transfer can induce protective immunity will also identify candidate antigens for vaccine development. Evidence that inability to express an antigen due to mutation is associated with avirulence is another useful criteria for antigen selection especially if the antigen is surfacelocalized or secreted.

Many protective antigens gain access to antigen-presenting pathways, and one can employ genomics mining with algorithms to identify subsets of proteins that are secreted by various secretion pathways or are surface-localized. For example, antigens delivered by the type III secretion system of various pathogens such as Salmonella and Yersinia are delivered to the cytoplasm of host cells to result in CD8-restricted CTL responses (12). This result may also be true for proteins secreted by other pathogens such as Mycobacterium species, if these proteins are secreted into the cytoplasm of cells within the infected individual. Also, if a surface antigen from one pathogen is found to induce protective immunity, one can search by computer for homologs in other pathogens that can be evaluated for their ability to induce protective immune responses.

There is a possibility that given protective antigen is synthesized only in vivo, with the gene encoding it responding to some stress or signal. Such antigens are not expressed during in vitro cultivation. Genetic screens such as In Vivo Expression Technology (IVET) (44), Signature-Tagged Mutagenesis (STM) (45), and
Selective Capture of Transcribed Sequences (SCOTS) (46) can be useful in identifying in vivo-expressed genes encoding proteins essential for survival of the pathogen in vivo. Proteins constitutively synthesized by recombinant clones of genes uniquely expressed in vivo can then be used to recognize either antibodies or lymphocyte populations responding to that antigen. Proteomic analysis, quantitating proteins synthesized when the pathogen is in an in vivo compartment or in cells in culture, can identify proteins synthesized in the greatest amounts. Such proteins have a higher likelihood of inducing immune responses may or may not be protective.

\section{Biotechnology and vaccine design}

Most articles describing design of recombinant attenuated vaccines omit mention of biotechnological means to improve the performance of the recombinant attenuated antigen delivery vaccine. One significant exception is the consideration that has been given to the promoter sequence used to drive expression of a plasmid-encoded protective antigen. In this regard, use of the nirB promoter, which functions optimally under the anaerobic conditions encountered in vivo, has given very promising results with recombinant attenuated Salmonella vaccine constructs (5). Often ignored among vaccine properties, codon usage patterns have speciesspecific differences, and codons in genes specifying an antigen from a heterologous species often need to be optimized for high-level expression in the vaccine strain. It may also be desirable to add DNA sequences specifying $5^{\prime}$ and $3^{\prime}$ untranslated mRNA sequences that create stem-loop structures and to remove potential targets within the coding mRNA sequences to minimize mRNA degradation by the mRNA degradosome (47). Deletion of the gene for poly(A) polymerase I can also increase mRNA stability (48) to possibly improve the productive fitness of the recombinant vaccine strain.

The stability of the synthesized antigen in the bacterial antigen delivery strain must be considered, in order to preclude toxicity of breakdown products as well as, after delivery to the immunized host, to enhance induction of the desired type of immune response. Thus, $\mathrm{T}$ cell antigens, which must be degraded by the proteosome prior to antigen presentation, might benefit from changes that decrease their stability. Conversely, antigens to which a humoral immune response is desired should be engineered for greater stability in the immunized host. Attention to the $\mathrm{N}$-end rule for protein degradation (49) and the presence of PEST sequences (50) and other protease cleavage sites in the antigen can help in design considerations. If the foreign antigen contains cysteine residues, one must be concerned about anomalous protein folding, which can be rectified by deletion of Cys-encoding sequences, changing of the environment of the antigen delivery strain with regard to Eh potential, inclusion of a chaperone for the expressed antigen, or modification of the $d s b$ system for isomerization of proteins with disulfide bonds (51). Another means to enhance immunogenicity of an expressed protective antigen might be inclusion of 
sequences leading to lipidation, since lipoproteins can be taken up more efficiently by antigen-presenting cells to induce an array of desired immune responses (52). Since expression of proteins with extensive hydrophobic domains very often results in toxicity to the antigen delivery vector, deleting of these sequences should be considered, since immune responses to them are seldom protective. Fusion of proteins to facilitate the secretion of protective antigens $(3,11)$, or their delivery to the cytoplasm by type III secretion systems to stimulate $\mathrm{T}$ cell immunity (12), has been considered in several cases. Such fusions can also be used to stabilize proteins or to enhance induction of immune responses by the fusion partner encoding a strong or universal epitope. Coexpression of cytokines along with antigenic sequences might be helpful as well, since many of these factors can be synthesized and secreted in a bioactive form from recombinant attenuated bacterial antigen delivery strains.

\section{Evaluation in animals prior to human testing}

Attaining the ultimate goal of vaccines that are safe and efficacious in humans can be both advanced and hindered by studies in animal systems. For example, the use of S. typhimurium in mice as a surrogate for $S$. typhi in humans has many real advantages. On the other hand, the disease caused by S. typhimurium in mice is not exactly the same as that caused by $S$. typhi infection in humans. To make matters worse, most scientists rely on mice that are inbred rather than outbred and that possess unique susceptibility to $S$. typhimurium infection. Similarly, use of outbred mice to evaluate candidate recombinant attenuated S. typhi vaccines is likely very misleading. Evaluation of $S$. typhi attenuation has often employed intraperitoneal inoculation of outbred mice in the presence of hog gastric mucin (53). This assay seems to have worked very well for attenuated strains with aro mutations that preclude growth of the S. typhi but would lead one to believe that bacteria with $p h o P Q$ deletions are as virulent as wild-type bacteria, since their growth is not impeded and the mice succumb to endotoxic shock after growth of the S. typhi strain (54). It is also well known that $S$. typhi is unable to survive in murine macrophages of diverse types, and therefore the immunogenicity of $S$. typhi constructs after intranasal inoculation into mice is probably no different from that achieved by a diversity of pathogens that are unable to cause lethal infection in mice regardless of whether attenuating mutations are present. The development of strains of mice with enhanced susceptibility to $S$. typhi might overcome these problems. In this regard, the recent generation of transgenic mice with a receptor allowing $L$. monocytogenes infection via oral inoculation (55) might provide a better model for development of recombinant attenuated L. monocytogenes vaccines for humans. Work toward development of improved M. tuberculosis-derived recombinant vaccines necessitates the discovery of attenuated M. tuberculosis strains unable to establish latent infections. Nevertheless, results from tests of vaccine candidates in mice or guinea pigs probably will not be reflective of responses in humans. Therefore, more studies on these various vaccine candidates must be conducted with human volunteers.

\section{Conclusion}

Progress in the development of attenuated bacterial vaccines to control diseases caused by those bacterial pathogens in humans is encouraging. The ultimate benefit, however, is likely to be achieved by the use of some of these attenuated bacterial vaccines, or modified derivatives thereof, to express antigens from other pathogens to induce protective immunity to those pathogens. Use of live recombinant attenuated bacterial antigen delivery systems would be akin to immunization of the individual with a vaccine factory, since production of the protective antigen and its presentation to the immunized individual occur in vivo after immunization. Such vaccines are inexpensive to manufacture and can be lyophilized to be reconstituted at the time of use, thus likely avoiding the necessity of refrigeration. Their oral or intranasal administration eliminates the need for reliance on syringes with their inherent added cost and association with accessory unwanted hazards. Potential problems associated with repeated use of the same or similar antigen delivery vectors for the delivery of different protective antigens require further careful study and might necessitate modification of surface antigen attributes on the antigen delivery vectors.

\section{Acknowledgments}

I thank Josephine E. Clark-Curtiss, Richard Groger, Ho Young Kang, and Joel Dworkin for helpful suggestions to improve the manuscript. I thank Erika Arch, Wendy Bollen, and Xin Zhang for help with reference collection and manuscript preparation. Research has been supported by grants from the NIH, the US Department of Agriculture, and Bristol-Myers Squibb Co.

1. Nakayama, K., Kelly, S.M., and Curtiss, R., III. 1988. Construction of an $\mathrm{Asd}^{+}$expression-cloning vector: stable maintenance and high level expression of cloned genes in a Salmonella vaccine strain. Biotechnology (NY). 6:693-697.

2. Galen, J.E., et al. 1999. Optimizing of plasmid maintenance in the attenuated live vector vaccine strain Salmonella typhi CVD 908-htrA. Infect. Immun. 67:6424-6433.

3. Kang, H.Y., Srinivasan, J., and Curtiss, R., III. 2002. Immune responses to recombinant pneumococcal PspA antigen delivered by live attenuated Salmonella enterica serovar Typhimurium vaccine. Infect. Immun. 70:1739-1749.

4. Curtiss, R., III. 1990. Attenuated Salmonella strains as live vectors for the expression of foreign antigens. In New generation vaccines. G.C. Woodrow and M.M. Levine, editors. Marcel Dekker Inc. New York, New York, USA. $161-188$.

5. Roberts, M., Li, J., Bacon, A., and Chatfield, S. 1998. Oral vaccination against tetanus: comparison of the immunogenicities of Salmonella strains expressing fragment $C$ from the nirB and $b$ tr $A$ promoters. Infect. Immun. 66:3080-3087.

6. Dunstan, S.J., Simmons, C.P., and Strugnell, R.A. 1999. Use of in vivoregulated promoters to deliver antigens from attenuated Salmonella enterica var. Typhimurium. Infect. Immun. 67:5133-5144.

7. Orr, N., Galen, J.E., and Levine, M.M. 2001. Novel use of anaerobically induced promoter, $d m s A$, for controlled expression fragment $\mathrm{C}$ of tetanus toxin in live attenuated Salmonella enterica serovar typhi strain CVD 908-htrA. Vaccine. 19:1694-1700.

8. Guzman, L.-M., Belin, D., Carson, M.J., and Beckwith, J. 1995. Tight regulation, modulation, and high-level expression by vectors containing the arabinose $\mathrm{P}_{\mathrm{BAD}}$ promoter. J. Bacteriol. 177:4121-4130. 
9. Roberts, M., Chatfield, S.N., and Dougan, G. 1994. Salmonella as carriers of heterologous antigens. In Novel delivery systems for oral vaccines. D.T. O’Hagan, editor. CRC Press. Boca Raton, Florida, USA. 27-58.

10. Hess, J., et al. 2000. Protection against murine tuberculosis by an attenuated recombinant Salmonella typhimurium vaccine strain that secretes the 30-kDa antigen of Mycobacterium bovis BCG. FEMS Immunol. Med. Microbiol. 27:283-289.

11. Hess, J., et al. 2000. Secretion of different listeriolysin cognates by recombinant attenuated Salmonella typhimurium: superior efficacy of haemolytic over non-haemolytic constructs after oral vaccination. Microbes Infect. 2:1799-1806.

12. Rüssmann, H., et al. 1998. Delivery of epitopes by the Salmonella Type III secretion system for vaccine development. Science. 281:565-568.

13. Galen, J.E., and Levine, M.M. 2001. Can a 'flawless' live vector vaccine strain be engineered? Trends Microbiol. 9:372-376.

14. Behr, M.A., et al. 1999. Comparative genomics of BCG vaccines by wholegenome DNA microarray. Science. 284:1520-1523.

15. Levine, M.M., et al. 1999. Duration of efficacy Ty21a, attenuated Salmonella typhi live oral vaccine. Vaccine. 17(Suppl. 2):S22-S27.

16. Hone, D., et al. 1988. A galE via (Vi antigen-negative) mutant of Salmonella typhi $\mathrm{Ty} 2$ retains virulence in humans. Infect. Immun. 56:1326-1333.

17. Bumann, D., Hueck, C., Aebischer, T., and Meyer, T.F. 2000. Recombinant live Salmonella spp. for human vaccination against heterologous pathogens. FEMS Immunol. Med. Microbiol. 27:357-364.

18. Tacket, C.O., et al. 1992. Comparison of the safety and immunogenicity of $\Delta$ aroC $\Delta$ aroD and $\Delta$ cya $\Delta$ crp Salmonella typhi strains in adult volunteers. Infect. Immun. 60:536-541.

19. Chatfield, S.N., et al. 1992. Evaluation of Salmonella typhimurium strains harboring defined mutations in $h t r A$ and $\operatorname{aro} A$ in the murine salmonellosis model. Microb. Pathog. 12:145-151.

20. Roberts, M., Chatfield, S., Pickard, D., Li, J., and Bacon, A. 2000. Comparison of abilities of Salmonella enterica serovar Typhimurium aroA aroD and $\operatorname{aro} A$ btrA mutants to act as live vectors. Infect. Immun. 68:6041-6043.

21. Tacket, C.O., et al. 2000. Phase 2 clinical trial of attenuated Salmonella enterica serovar Typhi oral live vector vaccine CVD 908-btrA in U.S. volunteers. Infect. Immun. 68:1196-1201.

22. Hohmann, E.L., Oletta, C.A., Killeen, K.P., and Miller, S.I. 1999. phoP/phoQ-deleted Salmonella typhi (Ty800) is a safe and immunogenic single-dose typhoid fever vaccine in volunteers. J. Infect. Dis. 173:1408-1414.

23. Shlyakhov, E.N., and Rubinstein, E. 1993. Human live anthrax vaccine in the former USSR. Vaccine. 12:727-730.

24. Barnard, J.P., and Friedlander, A.M. 1999. Vaccination against anthrax with attenuated recombinant strains of Bacillus anthracis that produce protective antigen. Infect. Immun. 67:562-567.

25. Cohen, S., et al. 2000. Attenuated nontoxinogenic and nonencapsulated recombinant Bacillus anthracis spore vaccines protect against anthrax. Infect. Immun. 68:4549-4558.

26. Coster, T.S., et al. 1999. Vaccination against shigellosis with attenuated Shigella flexneri 2a strain SC602. Infect. Immun. 67:3437-3443.

27. Kotloff, K.L., et al. 2000. Shigella flexneri 2a strain CVD 1207, with specific deletions in $\operatorname{vir} G$, sen, set and guaBA, is highly attenuated in humans. Infect. Immun. 68:1034-1039.

28. Ryan, E.T., and Calderwood, S.B. 2000. Cholera vaccines. Clin. Infect. Dis. 31:561-565.

29. Svanborg, C., Orskov, F., and Orskov, I. 1994. Fimbriae and disease. In Fimbriae adhesion, genetics, biogenesis, and vaccines. P. Klemm, editor. CRC Press. Boca Raton, Florida, USA. 239-254.

30. Ohara, N., and Yamada, T. 2001. Recombinant BCG vaccines. Vaccine. 19:4089-4098

31. Nardelli-Haefliger, D., et al. 1996. Oral and rectal immunization of adult female volunteers with a recombinant attenuated Salmonella typhi vaccine strain. Infect. Immun. 64:5219-5224.

32. Tacket, C.O., et al. 1997. Safety and immunogenicity in humans of an attenuated Salmonella typhi vaccine vector strain expressing plasmidencoded hepatitis B antigens stabilized by the Asd-balanced lethal vector system. Infect. Immun. 65:3381-3385.

33. DiPetrillo, M.D., Tibbetts, T., Kleanthous, H., Killeen, K.P., and Hohmann, E.L. 1999. Safety and immunogenicity of $p h o P / p h o Q$-deleted Salmonella typhi expressing Helicobacter pylori urease in adult volunteers. Vaccine. 18:449-459.

34. Nickerson, C.A., and Curtiss, R., III. 1997. Role of sigma factor RpoS in initial stages of Salmonella typhimurium infection. Infect. Immun. 65:1814-1823.

35. Coynault, C., and Norel, F. 1999. Comparison of the abilities of Salmonella typhimurium rpoS, aroA and rpoS aroA strains to elicit humoral immune responses in BALB/c mice and to cause lethal infection in athymic BALB/c mice. Microb. Pathog. 26:299-305.

36. Gómez-Duarte, O.G., et al. 1995. Expression of fragment C of tetanus toxin fused to a carboxyl-terminal fragment of diphtheria toxin in Salmonella typhi CVD 908 vaccine strain. Vaccine. 13:1596-1602.

37. Tacket, C.O., et al. 2000. Safety and immune responses to attenuated Salmonella enterica serovar Typhi oral live vector vaccines expressing tetanus toxin fragment C. Clin. Immunol. 97:146-153.

38. Orr, N., Galen, J.E., and Levine, M.M. 1999. Expression and immunogenicity of a mutant diphtheria toxin molecule, $\mathrm{CRM}_{197}$, and its fragments in Salmonella typhi vaccine strain CVD 908-htrA. Infect. Immun. 67:4290-4294.

39. Edelman, R., et al. 1998. Safety and immunogenicity of recombinant Bacille Calmette-Guérin (rBCG) expressing Borrelia burgdorferi outer surface protein A (OspA) lipoprotein in adult volunteers: a candidate Lyme disease vaccine. Vaccine. 17:904-914.

40. Horwitz, M.A., Harth, G., Dilon, B.J., and Maslesa-Galic, S. 2000. Recombinant Bacillus Calmette-Guérin (BCG) vaccines expressing the Mycobacterium tuberculosis 30-kDa major secretory protein induce greater protective immunity against tuberculosis than conventional BCG vaccines in a highly susceptible animal model. Proc. Natl. Acad. Sci. USA. 97:13853-13858.

41. Gunn, G.R., et al. 2001. Two Listeria monocytogenes vaccine vectors that express different molecular forms for human papilloma virus-16 (HPV-16) E7 induce qualitatively different T cell immunity that correlates with their ability to induce regression of established tumors immortalized by HPV-16. J. Immunol. 167:6471-6479.

42. Mesnage, S., Weber-Levy, M., Haustant, M., Mock, M., and Fouet, A. 1999. Cell surface-exposed tetanus toxin fragment $C$ produced by recombinant Bacillus anthracis protects against tetanus toxin. Infect. Immun. 67:4847-4850.

43. Altboum, Z., Barry, E.M., Losonsky, G., Galen, J.E., and Levine, M.M. 2001. Attenuated Shigella flexneri 2a $\triangle$ guaBA strain CVD 1204 expressing enterotoxigenic Escherichia coli (ETEC) CS2 and CS3 fimbriae as a live mucosal vaccine against Shigella and ETEC infection. Infect. Immun. 69:3150-3158.

44. Mahan, M.J., Slauch, J.M., and Mekalanos, J.J. 1993. Selection of bacterial virulence genes that are specifically induced in host tissues. Science. 259:686-688.

45. Hensel, M., et al. 1995. Simultaneous identification of bacterial virulence genes by negative selection. Science. 269:400-403.

46. Graham, J.E., and Clark-Curtiss, J.E. 1999. Identification of Mycobacterium tuberculosis RNAs synthesized in response to phagocytosis by human macrophages by selective capture of transcribed sequences (SCOTS). Proc. Natl. Acad. Sci. USA. 96:11554-11559.

47. Liou, G.-G., Jane, W.-N., Cohen, S.N., Lin, N.-S., and Lin-Chao, S. 2001. RNA degradosomes exist in vivo in Escherichia coli as multicomponent complexes associated with the cytoplasmic membrane via the N-terminal region of ribonuclease E. Proc. Natl. Acad. Sci. USA. 98:63-68.

48. O'Hara, E.B., et al. 1995. Polyadenylylation helps regulate mRNA decay in Escherichia coli. Proc. Natl. Acad. Sci. USA. 92:1807-1811.

49. Varshavsky, A. 1996. The N-end rule: functions, mysteries, uses. Proc. Natl. Acad. Sci. USA. 93:12142-12149.

50. Rogers, S., Wells, R., and Rechsteiner, M. 1986. Amino acid sequences common to rapidly degraded proteins: the PEST hypothesis. Science. 234:364-368

51. Ritz, D., and Beckwith, J. 2001. Roles of thiol-redox pathways in bacteria. Annu. Rev. Microbiol. 55:21-48.

52. Baier, W., Masihi, N., Huber, M., Hoffmann, P., and Bessler, W.G. 2000. Lipopeptides as immunoadjuvants and immunostimulants in mucosal immunization. Immunobiology. 201:391-405.

53. Powell, C.J., DeSett, C.R., Lowenthal, J.P., and Berman, S. 1980. The effect of adding iron to mucin on the enhancement of virulence for mice of Salmonella typhi strain TY 2. J. Biol. Stand. 8:79-85.

54. Baker, S.J., Daniels, C., and Morona, R. 1997. PhoP/Q regulated genes in Salmonella typhi identification of melittin sensitive mutants. Microb. Pathog. 22:165-179.

55. Lecuit, M., et al. 2001. A transgenic model for Listeriosis: role of internalin in crossing the intestinal barrier. Science. 292:1665-1667. 